In Cres. Vol. $3 N^{\circ}$ 2: pp. 313-323, 2012

\title{
EFECTO DE LAS INFLORESCENCIAS DE BRÓCOLI SOBRE LA CAQUEXIA EN CÁNCER DE COLON INDUCIDO EN Rattus norvegicus albinus"
}

\author{
EFFECT OF THE INFLORESCENCES OF BROCCOLI \\ ON CACHEXIA IN COLON CANCER INDUCED \\ IN Rattus norvegicus albinus
}

\section{Luis Jesús Guzmán Velásquez, Suli Magali Irigoin López, Ana María Guevara Vasquez.2}

\begin{abstract}
RESUMEN
El objetivo de este estudio fue determinar el efecto de las inflorescencias de Brassica oleraceae italica "Brócoli" sobre la caquexia en cáncer de colon inducido en Rattus norvegicus albinus. Se trabajó con 18 especímenes machos adultos, divididos aleatoriamente en tres grupos de seis especímenes cada uno: control, blanco y problema (que recibió $60 \mathrm{mg} / 100 \mathrm{~g}$ p.c de liofilizado de inflorescencias de $B$. oleraceae, vía oral). Se utilizó 1,2-Dimetilhidrazina (DMH) para inducir cáncer de colon en los grupos control y problema a la dosis de $21 \mathrm{mg} / \mathrm{kg}$ p.c/ semana, vía subcutánea durante 18 semanas. Las ratas albinas fueron pesadas semanalmente durante ocho meses, obteniéndose un promedio del peso corporal mensual por espécimen de cada grupo y el promedio mensual por grupo de estudio. Los parámetros que se consideraron para evaluar la caquexia fueron: adelgazamiento, astenia, alopecia incipiente y xerosis conjuntival; asimismo los resultados muestran evidente pérdida de peso corporal en el grupo control y mínima pérdida en el grupo que recibió Brassica oleraeceae italica $\left(^{* *} \mathrm{p}<0,01\right)$. Se concluye que las inflorescencias de Brassica oleraceae itálica a la dosis de $60 \mathrm{mg} / 100 \mathrm{~g}$ p.c evita la pérdida de peso corporal y disminuye la afectación caquéctica en Rattus norvegicus albinus con cáncer de colon inducido con 1,2-dimetilhidrazina.
\end{abstract}

PALABRAS CLAVE: Brassica oleraceae italica "Brócoli", cáncer de colon, caquexia, 1,2dimetilhidrazina, Rattus norvegicus albinus.

* Recibido: 04 de mayo del 2012; aprobado: 25 de octubre del 2012.

1 Químico Farmacéutico. Universidad Nacional de Trujillo.

2 Químico Farmacéutico. Doctora en Ciencias Biomédicas, Docente Universidad Nacional de Trujillo. 


\begin{abstract}
The aim of this study was to determine the effect of the inflorescences of Brassica oleraceae italica on cachexia in colon cancer induced in Rattus norvegicus albinus. Specimens were 18 adult males albino rats, randomly divided into three groups of six specimens each: control, white and problem (which received $60 \mathrm{mg} / 100 \mathrm{~g}$ bw of lyophilized B. oleraceae inflorescences, orally). We used 1,2-dimethylhydrazine (DMH) to induce colon cancer in the control and problems groups at a dose of $21 \mathrm{mg} / \mathrm{kg}$ bw / week, subcutaneously for 18 weeks. The parameters considered to assess cachexia were: weight loss, asthenia, alopecia incipient and conjunctival xerosis, likewise the results show evident loss of body weight in the control group and minimal loss in the group receiving $B$. oleraceae $(* * p<0.01)$. We conclude that the inflorescences of Brassica oleraceae italic at the dose of $60 \mathrm{mg} / 100 \mathrm{~g} \mathrm{bw}$ prevents loss of body weight and decreased involvement in Rattus norvegicus cachectic albinus colon cancer induced by 1,2-dimethylhydrazine.
\end{abstract}

KEY WORDS: Brassica oleraceae italica "Broccoli", colon cancer, cachexia, 1,2dimethylhydrazine, Rattus norvegicus albinus.

\title{
I. INTRODUCCIÓN
}

La caquexia es un síndrome consuntivo presente en más de dos terceras partes del conjunto de pacientes que mueren de cáncer avanzado. Asimismo, este síndrome también aparece asociado a otros estados patológicos, como pueden ser las infecciones crónicas o los traumatismos de distinta índole. La caquexia se caracteriza por una importante y progresiva pérdida de peso corporal, así como por anorexia, astenia, anemia, náuseas crónicas e inmunosupresión. De éstas, la pérdida de peso corporal es una de las más aparentes, y es atribuible principalmente a una disminución de la masa muscular y adiposa, las cuales pueden llegar a representar entre un $20 \%$ a $40 \%$ de los fallecimientos asociados al cáncer. ${ }^{1,2}$

Clínicamente, la importancia de la caquexia es considerable, por cuanto existe una clara correlación inversa entre el grado de caquexia y la supervivencia del paciente. Además, la caquexia implica siempre una prognosis desfavorable, una menor respuesta a la terapia y una disminución en la calidad de vida del paciente. En función del tipo de tumor, su incidencia puede variar entre un $20 \%$ y un $80 \% .^{3}$

Los orígenes de la caquexia han de buscarse en dos aspectos fundamentales: una incrementada demanda calórica ocasionada por la presencia del tumor y la malnutrición debido a la anorexia. Dichos cambios están asociados a la presencia de diferentes factores solubles como citocinas producidas por el tumor y por huésped en respuesta al primero. ${ }^{2}$ 
La anorexia es un componente casi universal de la caquexia. Resulta poco probable que la anorexia sea por sí sola responsable del desgaste que se observa en los pacientes cancerosos, aunque puede ser un factor que contribuya a dicho desgaste. Los pacientes con cáncer gástrico, pancreático, colorrectal o pulmonar experimentan una significativa pérdida de peso, incluyendo disminución de la masa muscular esquelética, desarrollo de caquexia con o sin anorexia. Se conoce que en el momento del diagnóstico el $80 \%$ de pacientes con cáncer al tracto gastrointestinal y el $60 \%$ de pacientes con cáncer de pulmón ya han experimentado una pérdida de peso significativa. ${ }^{4}$

Cáncer colorrectal es el cuarto diagnóstico de cáncer más frecuente y la segunda causa de muerte por cáncer en los Estados Unidos. En el 2010 se estimaron aproximadamente 102900 nuevos casos de cáncer de colon y se estima un aumento del $9 \%$ de nuevos casos para el 2012. En nuestra localidad, según el Instituto Regional de Enfermedades Neoplásicas - Norte, el cáncer colorrectal se ubica dentro de las seis primeras causas de ingresos registrados durante el 2008 al 2011. Su incidencia por sexos es similar, considerándose una afectación a partir de los 50 años de edad. ${ }^{4-8}$

La sintomatología del cáncer colorrectal básicamente se caracteriza por cambios en el hábito intestinal, sangre en heces, tenesmo, descarga de moco, síndrome anémico con microcitosis y ferropenia y afección del estado general con pérdida de peso. La progresión local de la enfermedad puede producir obstrucción, especialmente en el sigma, hemorragias y perforaciones intestinales. La disminución progresiva de la función de este órgano afectado asociado a una variedad de complicaciones sistémicas de índole tumoral y humoral, es responsable del estado de malnutrición que cursa el paciente, empeorando notablemente el pronóstico de la enfermedad.

Brassica oleraceae italica "Brócoli" es un vegetal de la familia Brasicáceas, antes llamadas crucíferas. Esta planta posee abundantes cabezas florales carnosas de color verde, puestas en forma de árbol, sobre ramas que nacen de un grueso tallo comestible. Es un cultivo de clima fresco, cuyo origen probable se centra en las costas noreste del Mediterráneo. Así mismo, cultivada en el Perú, el potencial saludable se ha relacionado con su alta concentración de constituyentes bioactivos como flavonoides, carotenos y glucosinolatos, siendo esta concentración variable en función de la variedad, estado de madurez y tratamiento postcosecha. ${ }^{11,12}$

La alta tasa de mortalidad y la falta de una cultura de prevención en cáncer, y ante la carencia de estudios realizados sobre el efecto Brassica oleraceae itali- 
ca "Brócoli" en el cáncer de colon, motivó a la realización de esta investigación para que con los resultados obtenidos se proporcione un sustento científico que beneficie a la comunidad, ya que este vegetal está al alcance de la población en general.

\section{Problema}

¿Cuál es el efecto de las inflorescencias de Brassica oleraceae italica "Brócoli" sobre la caquexia en cáncer de colon inducido en Rattus norvegicus albinus?

\section{HIPÓTESIS}

Las inflorescencias de Brassica oleraceae italica "Brócoli" evita la pérdida de peso corporal y disminuye la afectación caquéctica en el modelo experimental de cáncer de colon en Rattus norvegicus albinus.

\section{OBJETIVo}

Determinar el efecto de las inflorescencias de Brassica oleraceae italica "Brócoli" sobre la caquexia en cáncer de colon inducido en Rattus norvegicus albinus.

\section{MATERIAL Y MÉTODO}

1. RECOLECCIÓN Y PREPARACIÓN DEL MATERIAL VEGETAL

a) Recolección y estabilización

Las inflorescencias de Brassica oleraceae itálica "Brócoli" fueron recolectadas en el centro poblado de Tarmatambo, provincia Tarma, región Junín, y luego fueron identificadas en el Herbarium Truxillensis de la Universidad Nacional de Trujillo HUT. La muestra se lavó, desinfectó y estabilizó con alcohol etílico $70^{\circ}$.

b) Preparación del Liofilizado

Se pesaron $80 \mathrm{~g}$ de las inflorescencias, distribuyéndose en cuatro depósitos para liofilización, los cuales se llevaron a congelación durante 40 min y luego a liofilización por 14 horas hasta la formación de harina, ${ }^{12,13}$ obteniéndose el rendimiento para cálculo de dosis.

\section{c) Animales de experimentación}

Se utilizaron 18 especímenes de Rattus novergicus albinus, machos adultos, desparasitados y con peso promedio $200 \mathrm{~g}$ que fueron adquiridos en el Insti- 
tuto Nacional de Salud, Chorrillos, Lima, y alojados en el Bioterio de la Facultad de Farmacia y Bioquímica de la Universidad Nacional de Trujillo bajo condiciones de ciclo luz/ oscuridad de 12/12 horas, temperatura ambiental entre 22 y $24{ }^{\circ} \mathrm{C}$ y alimentados con dieta estándar y agua ad libitum. Todos los procedimientos fueron realizados acorde a los protocolos aprobados por la Institución y la Guía para el Cuidado y Uso de animales de laboratorio (NRC, USA 1996).

\section{MÉTODOS}

a) Inducción de cáncer de colon con 1,2-dimetilhidrazina y administración del liofilizado de las inflorescencias de Brassica oleraceae italica "Brócoli” en Rattus norvegicus albinus.

Se trabajó con tres grupos: un grupo blanco, un grupo control y un grupo problema, constituido por 6 especímenes de Rattus norvegicus albinus, cada uno distribuídos en forma aleatoria.

Grupo I (Blanco). Conformado por 6 ratas albinas, las cuales no recibieron tratamiento alguno; sólo la solución salina fisiológica una vez por semana durante 32 semanas. Fueron alimentados con dieta estándar y agua ad libitum.

\section{Inducción de cáncer de colon con 1,2-dimetilhidrazina ${ }^{6}$}

Las ratas fueron inyectadas semanalmente con 1,2-dimetilhidrazina $(\mathrm{DMH})$, subcutáneamente en la zona lumbar izquierda a la dosis de $21 \mathrm{mg} / \mathrm{kg}$ de peso corporal. La DMH fue disuelta en solución salina fisiológica $(10 \mathrm{mg} / \mathrm{ml})$.

Grupo II (Control). Conformado por 6 ratas, a las que se inyectó semanalmente una dosis DMH (21 mg/kg de peso, por vía subcutánea), desde el principio del estudio, durante 18 semanas. Fueron alimentados con dieta estándar y agua ad libitum hasta la semana 32 en que finalizó el estudio.

Grupo III (Problema). Grupo conformado por 6 ratas, a las que se inyectó DMH a la dosis de $21 \mathrm{mg} / \mathrm{kg}$ de peso, durante 18 semanas, por vía subcutánea, desde el principio del estudio y luego, a partir de la semana 19, se administró el liofilizado de las inflorescencias de Brassica oleraceae itálica "Brócoli", teniendo en cuenta el cálculo del rendimiento obtenido previamente, administrándose por vía oral la dosis de $60 \mathrm{mg} / 100 \mathrm{~g}$ de p.c. hasta la semana 32. También fueron alimentados con dieta estándar y agua ad libitum durante el tiempo que abarcó el estudio.

b) Comprobación del efecto de las inflorescencias de Brassica oleraceae italica "Brócoli" en Rattus norvegicus albinus. 
Los especímenes de todos los grupos de estudio fueron pesados semanalmente durante ocho meses, obteniéndose un promedio del peso corporal mensual por espécimen de cada grupo y el promedio mensual por grupo de estudio. Los parámetros que se consideraron para evaluar la caquexia fueron: adelgazamiento, astenia, alopecia incipiente y xerosis conjuntival, considerándose que $(+)$ significa que presenta muy leve afectación, $(++)$ leve afectación, $(+++)$ moderada afectación, $(++++)$ severa afectación y $(-)$ no presenta afectación.

\section{ANÁLISIS ESTADÍSTICO}

Los datos de pesos corporales fueron expresados como promedio y desviación estándar y analizados estadísticamente tomando como parámetro central la media aritmética. Se calculó la desviación error estándar y el estudio comparativo se realizó mediante la distribución t de Student. ${ }^{14}$ Para inferir estadísticamente en base a los resultados se consideró un nivel de significancia de 0,05 .

\section{RESULTADOS}

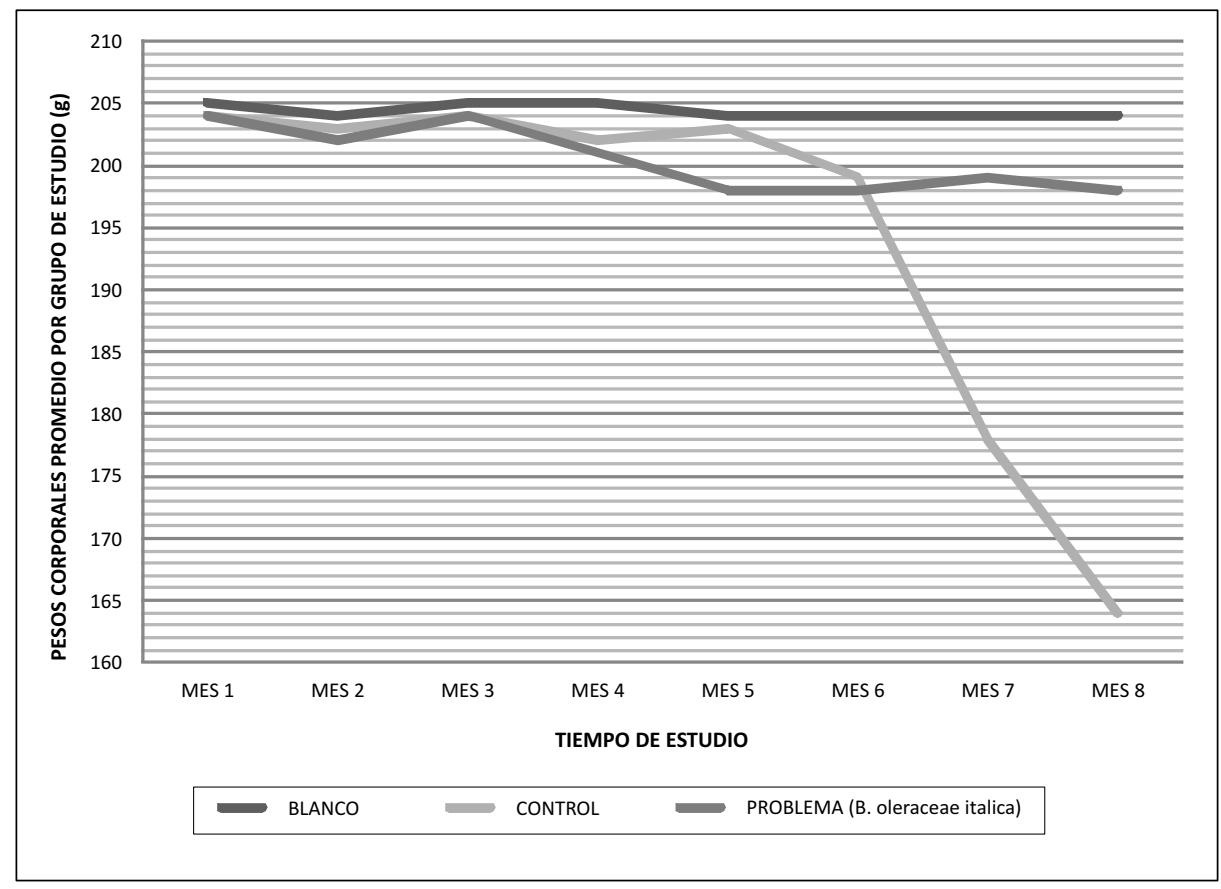

Figura 1. Variación de los pesos corporales promedio mensuales de Rattus norvegicus albinus de los grupos blanco, control y problema (Brassica oleraceae italica). 
Efecto de las inflorescencias de brócoli sobre la caquexia en cáncer de colon

Tabla 01

MANIFESTACIONES MÁS RELEVANTES DE LA AFECTACIÓN CAQUÉCTICA

EN Rattus norvegicus albinus

\begin{tabular}{lcccc}
\hline \multirow{2}{*}{ Grupos } & \multicolumn{4}{c}{ Caquexia en Rattus norvegicus albinus: } \\
\cline { 2 - 5 } & Adelgazamifestaciones mas relevantes \\
\hline BLANCO & - & Astenia & $\begin{array}{c}\text { Alopecia } \\
\text { incipiente }\end{array}$ & $\begin{array}{c}\text { Xerosis } \\
\text { conjuntival }\end{array}$ \\
CONTROL & ++++ & +++ & ++ & - \\
$\begin{array}{l}\text { PROBLEMA } \\
(\text { B. oleraceae italica })\end{array}$ & + & - & - & ++ \\
\hline
\end{tabular}

(-): no presenta manifestaciones de caquexia; $(+)$ : presenta muy leve afectación

$(++)$ : presenta leve afectación; $(+++)$ :presenta moderada afectación;

$(++++)$ : presenta severa afectación

Tabla 02

PESOS CORPORALES PROMEDIOS MENSUALES DE Rattus norvegicus albinus A PARTIR DEL MES DONDE ES MÁS EVIDENTE LA CAQUEXIA-ANOREXIA EN CÁNCER DE COLON

\begin{tabular}{lcccc}
\hline \multirow{2}{*}{ Grupos } & \multicolumn{4}{c}{ Pesos corporales promedio mensual (g) } \\
\cline { 2 - 5 } & MES 5 & MES 6 & MES 7 & MES 8 \\
\hline BLANCO & $204 \pm 2,25$ & $204 \pm 0,82$ & $204 \pm 1,87$ & $204 \pm 0,75$ \\
CONTROL & $203 \pm 1,52$ & $199 \pm 1,79 *$ & $178 \pm 5,69$ & $164 \pm 7,42^{* *}$ \\
PROBLEMA & & & & \\
$($ B. oleraceae italica $)$ & $198 \pm 2,48^{*}$ & $198 \pm 2,53$ & $199 \pm 3,5$ & $198 \pm 3,27 * *$ \\
\hline
\end{tabular}

Los valores expresan el promedio \pm DSM de $\mathrm{n}=6$ ratas albinas; ${ }^{*} \mathrm{p}<0,05, * * \mathrm{p}<0,01$

\section{DISCUSIÓN}

El cáncer es un problema mundial de salud pública; se le atribuyen 7,6 millones de defunciones ocurridas en 2008 (aproximadamente un 13\% del total). La mayor parte de la mortalidad anual por cáncer obedece a cáncer de pulmón, estómago, hígado, colon y mama. Una persona a los 50 años presenta un riesgo de un 5\% de padecer cáncer de colon antes de los 80 años y un riesgo de 2,5\% de morir de él. ${ }^{11}$ Anorexia, pérdida de peso involuntaria, degradación tisular, mal pronóstico y finamente muerte caracterizan la caquexia del cáncer, una condición de malnutrición calórica proteica avanzada. La caquexia se origina secundariamente como resultado de una incapacidad funcional para ingerir $o$ utilizar nutrientes. ${ }^{15-18}$ 
En la figura 1 se observa la variación de pesos corporales promedio mensual de los ejemplares del grupo problema, quienes presentan un similar comportamiento respecto al grupo blanco. Así mismo, la caída de la recta de los pesos corporales del grupo control se asocia a un pérdida de peso en función del tiempo, debido al desbalance energético y metabólico de la patología ejemplificada. ${ }^{1,2,10}$

El proceso evolutivo de la patología asociado a la pérdida de peso corporal producto de la inducción experimental con 1,2-dimetilhidrazina que se evidencia a partir del quinto mes de estudio. Esta observación se fundamenta en la capacidad carcinogénica órgano selectiva, la respuesta de factores humorales de los tumores ejemplificados y la respuesta de los especímenes ante dichos tumores, producto de la exposición del carcinógeno. ${ }^{6,14}$ Además, la tabla 01 muestra las manifestaciones más relevantes del proceso caquéctico durante la carcinogénesis experimental, considerando al adelgazamiento y la astenia entre las afectaciones más relevantes dentro del proceso consuntivo. ${ }^{2,4,9,22}$ El síndrome caquéctico, una manifestación del cáncer avanzado, relaciona a la anorexia como una consecuencia de la actividad de diversas citocinas como el Factor de Necrosis Tumoral- $\alpha$ (TNF- $\alpha$ ), interleucina - 1 (IL-1), interleucina (IL-6), y el interferon- $\gamma($ IFN- $\gamma){ }^{25,26,28}$ Los altos niveles séricos de estos mediadores anorexígenos producen a largo plazo una inhibición de la alimentación al inhibir la regulación de leptina y neuropéptido $Y .{ }^{19-22}$

En la tabla 02 se observa el peso corporal promedio mensual a partir del quinto mes de estudio, donde se evidencia la evolución en la pérdida de peso corporal de los especímenes del grupo problema, siendo ésta estadísticamente muy significativa con respecto al grupo control $(* * p<0,01)$. Se atribuye este hallazgo a la actividad de bioactivos presentes en las inflorescencias de Brassica oleraceae italica. ${ }^{29,31,32}$ Los hallazgos encontrados muestran similitud a los reportes con las especies Curcuma longa, Zingiber officinale, Camellia sinensis, Armoracia rustinaca las cuales evidencian eficacia en el tratamiento de caquexia neoplásica. ${ }^{23-26}$

Comparando los pesos corporales promedio y las desviaciones estándar en el octavo mes de estudio del grupo control $(164 \pm 7,42 \mathrm{~g})$ con respecto al grupo problema $(198 \pm 3,27 \mathrm{~g})$, y al grupo blanco $(204 \pm 0,75 \mathrm{~g})$, se observa una diferencia altamente significativa $(* * \mathrm{p}<0,01)$. Esto evidencia que las inflorescencias de Brassica oleraceae itálica revierte significativamente la pérdida de peso durante el proceso patológico del modelo experimental en estudio. Este efecto se atribuiría al sulforafano contenido en las inflorescencias del vegetal. Sulfora- 
fano proviene de la glucorafanina presente en Brassica oleraceae itálica, que actuaría de manera indirecta como un potente inductor enzimático de fase 2 del metabolismo de xenobióticos. Esta respuesta celular acelera la detoxificación de electrófilos y especies reactivas de oxígeno, y por lo tanto protege a la célula de los mecanismo inflamatorios iníciales durante el proceso carcinogénico. Es decir, las inflorescencias de Brassica oleraceae itálica evitan la pérdida de peso corporal asociado a la caquexia por una disminución de la actividad inflamatoria ejercida por los mediadores celulares durante el desarrollo tumoral. ${ }^{6,29-35}$

\section{CONCLUSIONES}

Las inflorescencias de Brassica oleraceae itálica a la dosis de $60 \mathrm{mg} / 100 \mathrm{~g}$ p.c evita la pérdida de peso corporal y disminuye la afectación caquéctica en Rattus norvegicus albinus con cáncer de colon inducido con 1,2dimetilhidrazina.

\section{REFERENCIAS BIBLIOGRÁFICAS}

1 ARgilés J, Busquets S, LóPEZ-Soriano F, Figueras M. Fisiopatología de la caquexia neoplásica. Departamento de Bioquímica y Biología Molecular. Universidad de Barcelona. España. Nutr. Hosp. v.21 supl.3. mayo 2006.

2 Kumar V, Abbas A, Fausto N. Patología esctructural y funcional. $7^{\text {a }}$ ed. Ed. Elsevier. Madrid. España. 2007. Pp. 337;453.

3 Acharyya S, Ladner K, Nelsen ll; Damrauer J; Reiser P; Swoap S. Cancer cachexia is regulated by selective targeting of skeletal muscle gene products. J Clin Invest; 114(3): 370-8, agosto. 2004.

4 Fox K, BROOKS J, GANDRA S, MARKUS R, CHIOU C. Estimation of cachexia among cancer patients based on four definitions. Journal of oncology. Vol.9, may 2009.

5 Engstrom P, Arnoletti J, Benson A, Chan E, Cooper H. Colon Cancer. NCCN Clinical Practice Guidelines in Oncology. Version 3.2011. Feb.2011.

6 PiÑols C. Estudios de los cambios de la mucosa colónica de rata y cinética celular durante la carcinogénesis experimental inducida con 1,2-dimetilhidrazina. Universitat de Lleida. Salamanca. España. Pp.16;23-25.

7 InSTITUTO REGIONAL DE ENFERMEDADES NEOPLÁSICAS. Area de vigilancia epidemioloógica del cáncer. Registro Hospitalario de Cáncer I-semetre 2011. Pp. 6-8;25-27.

8 Siegel R, Naishadham D, Jemal A. Cancer statics, 2012. American Cancer Society. CA: A Cancer Journal for clinicians. 2012; 62: 10-29.

9 Santaria L, Contaldo F, Pasanisi F. Nutritional screening and early treatment of malnutrition in cancer patients. J Cachex Sarcopenia Muscle 2011;1:27-35.

10 Fuci A, Braunwald E, Kasper D, Hauser S, Longo D, Jameson J. Principios de Medicina interna. $17^{\text {a }}$ ed. Ed. McGraw-Hill Interamericana. México. 2008. Pp. 255;451. 
11 Mostacero J. Taxonomía de las fanerógamas útiles del Perú. Vol. I. Trujillo. Normas Legales S.A.C. Perú. 2002. 266-267.

12 NAVAS R. Cuantificación de sulfurafano procedente de glucorafanina en retoños de Brassica oleracea var. italica por cromatografía líquida de alta resolución. Universidad de San Carlos de Guatemala. 2004.

13 Maldonado R, Pacheco E. Curvas de deshidratación del brócoli (Brassica oleraceae L var. Italica) y coliflor (Brassica oleraceae L var. Botrytis L). Revista de la Facultad de Agronomía. 20(3) Caracas. 2003.

14 Wayne W, Daniel. Bioestadística. Base para el análisis da las ciencias de la salud. $4^{\mathrm{a}}$ ed. Limusa Wiley. México 2004. 41-42.

15 Silverio R, Laviano A, FAnelli F, Seelaender M. 1-carnitine and cancer cachexia: Clinical and experimental aspects. J Cachex Sarcopenia Muscle 2011;1:37-44.

16 Kenneth C, Fearon M. Cancer Cachexia and Fat-Muscle Physiology. N Engl J Med 2011; 365:565-567August 11, 2011.

17 Jeffrey S, Chamberlain P. Cachexia in Cancer - Zeroing in on Myosin. N Engl J Med 2004; 351:2124-2125November 11, 2004.

18 Nussinovitch U, SHOENFELD Y. Cachexia, malignancy and tumor necrosis factor alpha (TNF-alpha). Harefuah; 146(5): 349-51, 406, 2007 May.

19 Figueras M, Busquets S, CARbó N, Almendro V, Argilés J. Cancer cachexia results in an increase in TNF-alpha receptor gene expression in both skeletal muscle and adipose tissue. Int J Oncol; 27(3): 855-60, 2005 Sep.

20 Abbas A, Lichtman A, Pillai S. Inmunología celular y molecular. $6^{\text {a }}$ ed. Ed. Elsevier. Barcelona. España. 2008. pp. 273.

21 AKIO I. Cancer Anorexy-Cachexia Syndrome: Current Issues in Research and Management. CA: A Cancer Journal for Clinicians. Volume 52, Issue 2, March/April 2002, p: 72-91.

22 Evoy D, Fahey T, Lieberman M. Nutritional support of the cancer patient . CA: A Cancer Journal for Clinicians Volume 48, Issue 2, March/April 1998, Pages: 69-80. ROSENBLOOM R. Method for the treatment of caquexia. United states patent. Mar,29.2009. Irzuka N, Hazama S, Yoshimura K, Yoshino S, Tangoku A. Anticachectic effects on the natural herb Coptidis rhizoma and berberine on mice bearing colon 26/clone 20 adenocarcinoma. Department of Bioregulatory Function. Yamaguchi University School of Medicine. Int. j cancer. 99,286-291. 2002.

25 Woon L, Yun B, Kim M, PARK C, Song W. Phenolic compounds isolated from Psoralea corilyfolia inhibit IL-6 induced STAT3 activation. Bioindustrial Process Research center. Korea. Planta Med. April. 2012.

26 Chang J, Lee S, Park M, Hudson B, Rho M. Kansuinine A and Kansuinine B from Euphorbia kansui L. inhibit IL-6 induced STAT3 activation. Korea Research Institute of Bioscience and Biotechnology. Korea. Planta Med. 76; 1544-1549. March. 2010.

27 ARAVINDARAM K, YANG N. Anti inflammatory plant natural products for cancer therapy. Agricultural Biotechnology Research Center. Taiwan. Planta Med. 76; 1103-1117. January. 2010.

28 Prasad S, Phrommol K, Yadav V, Chaturvedl M. Targeting inflammatory pathways by flavonoids for prevention and treatment of cancer. Citokine Research Laboratory. The University of Texas. Planta Med. 76; 1044-1063. June. 2010. 
Gescher A, Gerhauser C. Cancer Chemopreventive Agents in Plants - A Continuing Challenge. Cancer Biomarkers and Prevention Group. Department of Cancer Studies and Molecular Medicine. University of Leicester. Leicester. U.K. Planta Medica. 74; 15231525. August 2008.

30 Zhang Y, Talalay P, Chо C, et al. A major inducer of anticarcingenc protective emzymes from broccoli: Isolation and elucidation of structure. Department of Pharmacology and Molecular science, The Johns Hopkins University School of medicine. Proc. Natl. Acad. Scie. USA. Vol 83, pp 2399-2403. March 2000.

31 ANTOCIEWICZ J, ZIOLKOWSKI W, KAR S, et al. Role of Reactive Oxygen Intermediates in Cellular Responses to Dietary Cancer Chemopreventive Agents. Department of Bioenergetics and Physiology of Exercise. Medical University of Gdansk. Gdansk. Poland. Planta medica. 74; 1570-1579. June 2008.

32 Zhang Y, Talalay P. Cancer preventive isothiocyanates: measurement of human exposure and mechanism of action. Department of chemopevention. Roswell Park Cancer Institute. USA. Fundamental and Molecular Mechanisms of Mutagenesis Research. 555:173-190, September 2004.

33 Zhang Y, TANG L. Selected isothiocyanates rapidly induce growth inhibition of cancer cell. Arizona Cancer Center and Department of Medicine. College of Medicine. University of Arizona. Molecular Cancer Therapeutics Research. 2:1045-1052. May 2003.

34 Hashem F, Motawea H, Elshabrawy A, et al. Myrosinase hydrolysates of Brassica oleraceae L. var. italica Plenck. National Research Centre. Faculty of Pharmacy. Cairo University. Cairo. Egypt. Planta Medica. 77; 2011.

35 MCWalter G, Higgins L, MCLellan, et al. Transcription factor Nrf2 is essential for induction of NAD (P)H:quinone oxidoreductase 1, glutathione S-transferases, and glutamate cysteine ligase by broccoli seeds and isothiocyanates. Biomedical Research Centre. Ninewells Hospital and Medical School. University of Dundee. Scotland. United Kingdom. The American Society for Nutritional Sciences. The journal of nutrition. 134; 3499-3506. December 2004. 